\title{
Cinética de la transferencia de masa durante la deshidratación osmótica de yacón (Smallanthus sonchifolius)
}

\author{
Cinética de transferência de massa durante a desidratação osmótica de yacón (Smallanthus sonchifolius)
}

Silvina MALDONADO ${ }^{1 \star}$, Julia Eleonora SANTAPAOLA ${ }^{1}$, Judith SINGH, Martín TORREZ1 ${ }^{1}$, Alejandra GARAY ${ }^{1}$

\section{Resumen}

El yacón (Smallanthus sonchifolius) es un tubérculo andino de vida útil muy corta bajo condiciones ambientales. Los objetivos de este trabajo fueron determinar: 1) la cinética de deshidratación osmótica de yacón, utilizando sacarosa como soluto; 2) el ajuste de la ecuación de Peleg a los datos experimentales; y 3) el coeficiente de difusión usando la ecuación de Hawkes y Flink. La fruta se peló y cortó en placas de $3 \times 3 \times 0,3 \mathrm{~cm}$. Se la deshidrató osmóticamente con solución de sacarosa al $40 \%(\mathrm{p} / \mathrm{p})$, hasta aw $=0,97$. El proceso se realizó a temperatura de $25^{\circ} \mathrm{C}$ y con agitación continua ( $\left.105 \mathrm{rpm}\right)$. Se determinó la pérdida de peso de las muestras, la ganancia de sólidos y la retención de agua. Los parámetros obtenidos para el ajuste de pérdida de agua y ganancia de sólidos son respectivamente: $\mathrm{k}_{1}: 8,2$ 0,1 y $\mathrm{k}_{2}: 0,53 \pm 0,06 ; \mathrm{k}_{1}: 234 \pm 8$ y $\mathrm{k}_{2}$ : $2,6 \pm 0,5$. La mayor transferencia de masa, tanto de agua como de soluto, ocurre durante los primeros 60 a 90 minutos de proceso, lográndose una ganancia media de sólidos de 9,5 [g.100 g ${ }^{-1} \mathrm{MF}$ ] y una pérdida de agua de 68,8 [g.100 g-1 MF]. Se puede asegurar que es posible aplicar satisfactoriamente el proceso de deshidratación osmótica en yacón como pre tratamiento de conservación.

Palabras clave: yacón; transferencia de masa; difusión; deshidratación; ósmosis.

\section{Resumo}

O yacón (Smallanthus sonchifolius) é um tubérculo andino de vida útil muito curta sob condições ambientais. Os objetivos deste trabalho foram determinar: 1) a cinética de desidratação osmótica do yacón, utilizando sacarose como soluto; 2) o ajuste da equação de Peleg aos dados experimentais; e 3) o coeficiente de difusão usando a equação de Hawkes e Flink. A fruta foi descascada e cortada em placas de $3 \times 3 \times 0,3 \mathrm{~cm}$. Foi desidratada osmoticamente usando uma solução de sacarose aos $40 \%(\mathrm{p} / \mathrm{p})$, até aw $=0,97$. O processo foi conduzido mantendo a temperatura constante em $25^{\circ} \mathrm{C}$ e com agitação orbital contínua ( $105 \mathrm{rpm}$ ). Determinou-se a perda de peso das mostras, o ganho de sólidos e a retenção de água. Os parâmetros obtidos para o ajuste de perda de água e ganho de sólidos foram respectivamente: $\mathrm{k}_{1}: 8,2 \pm 0,1 \mathrm{e}$ $\mathrm{k}_{2}: 0,53 \pm 0,06 ; \mathrm{k}_{1}: 234 \pm 8$ e $\mathrm{k}_{2}: 2,6 \pm 0,5$. A maior transferência de massa, tanto de água como de soluto, ocorre durante os primeiros 60 a 90 minutos de processo, conseguindo-se um ganho de sólidos médio de 9,5 g. $100 \mathrm{~g}^{-1} \mathrm{MF}$ e uma perda de água de $68,8 \mathrm{~g} .100 \mathrm{~g}{ }^{-1} \mathrm{MF}$. Pode-se assegurar que é possível aplicar satisfatoriamente o processo de desidratação osmótica em yacón como pré-tratamento de conservação.

Palavras-chave: yacón; transferência de massa; difusão; desidratação; osmose.

\section{Introducción}

La región andina es conocida por ser cuna de algunos cultivos de importancia mundial como la papa, el camote y los ajíes. Sin embargo, gran parte de las plantas alimenticias domesticadas por los pueblos andinos son poco conocidas internacionalmente, incluso en los mismos países andinos. Entre estas plantas se encuentran las raíces y los tubérculos andinos, un grupo de nueve especies que incluye: achira, ahipa, arracacha, maca mauka, oca, ulluco y yacón. Todos ellos son importantes en la alimentación del poblador andino rural, pero su aprovechamiento está restringido.

El yacón es una planta domesticada hace varios siglos por los pobladores de las culturas prehispánicas, aunque hasta hace poco tiempo se cultivaba en huertos caseros para autoconsumo.
Recientemente se han empezado a descubrir y difundir algunas de sus propiedades, por lo que se ha generado en la población un creciente interés por este cultivo.

La planta de yacón presenta un tubérculo de características particulares: tiene un agradable sabor dulce y debido a su alto contenido acuoso, deja una sensación refrescante después de consumirlo, razón por la cual es considerado como una fruta. Además contiene fructooligosacáridos, azúcares que no pueden ser digeridos directamente por el organismo humano, debido a la ausencia de las enzimas necesarias para su metabolismo. Los fructooligosacáridos no elevan el nivel de glucosa en la sangre y poseen pocas calorías, alrededor de 1520 cal.100 g ${ }^{-1}$, comparados con otros tubérculos como la papa 
(120 cal.100 g ${ }^{-1}$ ) (HERMANN; FREIRE; PAZOS, 1999). Estas propiedades han convertido al yacón en un recurso potencialmente importante.

Hasta la década pasada existía la idea de que el valor nutricional del yacón era muy bajo, por lo que se ha realizado muy poca investigación acerca de su cultivo y de las tecnologías de procesamiento y transformación que se requieren desarrollar para lograr su mejor aprovechamiento (SEMINARIO; VALDERRAMA; MANRIQUE, 2003).

Desde hace unos pocos años, en la localidad de Bárcena, provincia de Jujuy, se vienen realizando esfuerzos por reinsertar este cultivo entre los productores, habiéndose logrado aumentar el interés por este tubérculo. Si bien se han realizado también intentos por conservarlo o agregarle valor, los productos desarrollados han sido obtenidos en forma rudimentaria, a partir de técnicas artesanales que precisan ser optimizadas, adaptadas y/o re-estructuradas para lograr productos organolépticamente aceptables e higiénico-sanitariamente adecuados.

La deshidratación osmótica (DO) es un proceso ampliamente estudiado en los últimos años y usado principalmente como pre-tratamiento de muestras que luego son sometidas a congelación o a deshidratación térmica (HAWKES; FLINK, 1978; KOWALSKA; LENART, 2001; ERLE; SCHUBERT, 2001; LEWICKI PIOTR, 1998).

El fenómeno de DO implica la remoción de agua del alimento y el ingreso de sólido desde la solución hipertónica hacia el interior del alimento (KOWALSKA; LENART, 2001; PETROTOS; LAZARIDES, 2001; MAGEL; HASSABALLAH; MURPHY, 1983; RAOULT-WACK; LENART; GUILBERT, 1992). La velocidad y el grado de deshidratación de un material y los cambios en su composición química dependen del tipo y la concentración de soluto usado, del tamaño de la muestra y el soluto, de la relación fruta:jarabe, de la temperatura, del tiempo y del equipo usado para el proceso (CONWAY et al., 1983; PONTING et al., 1966).

Generalmente la velocidad de la DO aumenta al principio del proceso como resultado de las diferencias de presión osmótica y la menor resistencia a la transferencia de masa en esta etapa del proceso (HAWKES; FLINK, 1978; KOWALSKA; LENART, 2001; LAZARIDES; KATSANIDIS; NICKOLAIDIS, 1995; EL-AQUAR; MURR, 2003; PETROTOS; LAZARIDES, 2001; RAOULT-WACK; LENART; GUILBERT, 1992).

La aplicación de este tipo de tecnología simple y de bajo costo, debido entre otros al menor requerimiento de energía (LAZARIDES; KATSANIDIS; NICKOLAIDIS, 1995), brinda la posibilidad de preservar alimentos altamente perecederos, como el yacón, que además tienen cortos períodos de cosecha.

Se han aplicado diferentes modelos matemáticos para ajustar los resultados experimentales de procesos de DO, con el objeto de predecir el comportamiento de los productos alimenticios. Uno de los modelos más usados es la ecuación empírica propuesta por Peleg (PETROTOS; LAZARIDES, 2001; SACCHETTI; GIANOTTI; DALLA ROSA, 2001; CONWAY et al., 1983) Este modelo no describe el proceso físico de difusión.
El modelo más usado es el basado en la ley difusional de Fick. Este supone que la concentración de la solución permanece constante y que la resistencia externa es despreciable frente a la resistencia interna (HAWKES; FLINK, 1978; MAGEL; HASSABALLAH; MURPHY, 1983; RAOULT-WACK; LENART; GUILBERT, 1992). La primera condición se cumple la mayoría de las veces, sin embargo la segunda no siempre queda satisfecha sobre todo cuando, como en este caso, se trabaja en condiciones que pueden favorecer las altas viscosidades (altas concentraciones de solución y/o temperaturas bajas) (RAOULT-WACK; LENART; GUILBERT, 1992) En estos casos las características de la transferencia superficial (tanto para agua como para sólidos) pueden ser relativamente pobres. Esta es la razón del uso del coeficiente D como coeficiente total o global de transferencia de masa. También para este modelo se supone que la geometría de la muestra es simple como, por ejemplo, una placa infinita (SALVATORI; ALZAMORA, 2000; MAGEL; HASSABALLAH; MURPHY, 1983).

Debido a estas restricciones, los coeficientes de transferencia de masa obtenidos son coeficientes de difusión aparente o coeficientes de pseudodifusión, que a menudo dependen de las condiciones de la materia prima y del tipo de tratamiento (LAZARIDES; KATSANIDIS; NICKOLAIDIS, 1995; PALOU et al., 1993).

El objetivo de este trabajo fue determinar la cinética de deshidratación osmótica de yacón, utilizando sacarosa como soluto y evaluar el ajuste de los resultados experimentales a modelos matemáticos preestablecidos; como el basado en la ecuación de Fick y el modelo de Peleg.

\section{Materiales y métodos}

\subsection{Preparación de la muestra}

Se trabajó con yacón, producido en la localidad de Bárcena, provincia de Jujuy, Argentina. Los tubérculos se obtuvieron directamente del campo y se trasladaron al laboratorio en cajones de madera. Se refrigeraron a $10 \pm 1^{\circ} \mathrm{C}$, hasta el ensayo, tiempo generalmente no mayor a los 10 días.

Se seleccionaron los tubérculos por su grado de madurez, determinada en forma combinada a través de evaluación organoléptica, principalmente firmeza, y la determinación de ${ }^{\circ}$ Brix mediante un refractómetro digital (ABBE Quartz Bélgica). La fruta se peló y cortó en placas de $3 \times 3 \times 0,3 \mathrm{~cm}$, con una precisión de $0,01 \mathrm{~cm}$. Para la deshidratación osmótica se utilizó solución de sacarosa al $40 \%(\mathrm{p} / \mathrm{p})$.

\subsection{Protocolo experimental}

La relación fruta/jarabe fue la necesaria para lograr una $\mathrm{a}_{\mathrm{w}}=0,97$. Los cálculos se realizaron teniendo en cuenta el balance de masa y las ecuaciones de Norrish y Ross.

La deshidratación osmótica se llevó a cabo manteniendo la temperatura constante a $25 \pm 1{ }^{\circ} \mathrm{C}$ y con agitación orbital continua (105 rpm) en un shaker marca Vicking. El estudio se realizó evaluándose el comportamiento del sistema en tres repeticiones y cada repetición se realizó por triplicado. 
Durante el proceso de DO se extrajeron muestras a tiempos preestablecidos. Una vez extraídas, se secaron con papel absorbente para eliminar el exceso de impregnante superficial.

Se determinó la variación del peso de las muestras al $0,001 \mathrm{~g}$ a cada tiempo, utilizando una balanza analítica Denver Instruments Estados Unidos 2005. La incorporación de sólidos solubles, se determinó a partir de la medición de los ${ }^{\circ}$ Brix a cada tiempo de proceso.

\subsection{Análisis de la cinética de deshidratación osmótica}

Se calculó la pérdida de peso y la ganancia de sólidos. La pérdida de agua se determinó gravimétricamente, y se realizó balance de masa para verificar la concordancia de las determinaciones. Los parámetros evaluados se expresaron de la siguiente forma:

WR: reducción o pérdida de peso [g.100 g-1 MF]

WL: pérdida de agua [g agua. $100 \mathrm{~g}^{-1} \mathrm{MF}$ ]

SG: ganancia de sólidos [g sólidos. $100 \mathrm{~g}^{-1} \mathrm{MF}$ ]

en que:

MF: muestra fresca inicial

La determinación de la pérdida de peso (WR), ganancia de sólidos (SG) y pérdida de agua (WL) se efectuó a través de la evaluación de las relaciones (1), (2), (3) y (4).

$$
\begin{aligned}
& \mathrm{WR} \%=\frac{\mathrm{W}_{\mathrm{i}}-\mathrm{W}_{\mathrm{t}}}{\mathrm{W}_{\mathrm{i}}} \times 100 \\
& \mathrm{SG} \%=\frac{\mathrm{SS}_{\mathrm{i}}-\mathrm{SS}_{\mathrm{t}}}{\mathrm{W}_{\mathrm{i}}} \times 100
\end{aligned}
$$

$\mathrm{WL} \%=\mathrm{SG} \%+\mathrm{WR} \%$

$$
\mathrm{WL} \%=\frac{\mathrm{M}_{\mathrm{i}}-\mathrm{M}_{\mathrm{t}}}{\mathrm{W}_{\mathrm{i}}} \times 100
$$

en que:

$\mathrm{W}_{\mathrm{i}}=$ peso de la muestra inicial en $\mathrm{g}$

$\mathrm{W}_{\mathrm{t}}=$ peso de la muestra al tiempo $\mathrm{t}$ en $\mathrm{g}$

$\mathrm{SS}_{\mathrm{i}}=$ sólidos solubles iniciales $\left({ }^{\circ} \mathrm{Brix}\right)$ expresados en $\mathrm{g}$ sólidos. $100 \mathrm{~g}^{-1} \mathrm{MF}$

$\mathrm{SS}_{\mathrm{t}}=$ sólidos solubles al tiempo $\mathrm{t}\left({ }^{\circ} \mathrm{Brix}_{\mathrm{t}}\right)$ expresados en $\mathrm{g}$ sólidos. $100 \mathrm{~g}^{-1} \mathrm{MF}$

$\mathrm{M}_{\mathrm{i}}=$ humedad inicial expresada en $\mathrm{g} \mathrm{H}_{2} \mathrm{O} .100 \mathrm{~g}^{-1} \mathrm{MF}$

$\mathrm{M}_{\mathrm{t}}=$ humedad al tiempo t expresada en $\mathrm{g} \mathrm{H}_{2} \mathrm{O} .100 \mathrm{~g}^{-1} \mathrm{MF}$

\subsection{Modelado matemático y tratamiento estadístico}

Se emplearon dos modelos matemáticos para el estudio del proceso de deshidratación osmótica: el primero fue el modelo Fickiano estándar, usado para obtener el coeficiente de difusión. Para la determinación del coeficiente de difusión se graficó el parámetro concentración en función de la raíz cuadrada del tiempo y el coeficiente de difusión se determinó midiendo la pendiente de las rectas resultantes.

Esta ecuación, formulada por Hawkes y Flink (1978), ha sido presentada mediante distintas expresiones para definir el parámetro concentración; en este caso se usó la pérdida de agua (WL) resultando la Ecuación 5.

$$
\frac{\mathrm{WL}}{\mathrm{WL}_{\infty}}=2\left(\frac{\mathrm{Dt}}{\pi \mathrm{l}^{2}}\right)^{1 / 2}
$$

en que:

$\mathrm{D}=$ coeficiente de difusión $\left[\mathrm{cm}^{2} / \mathrm{s}\right]$

$\mathrm{t}=$ tiempo $[\mathrm{s}]$

$\mathrm{WL}_{\infty}=$ pérdida de agua en el equilibrio

$\mathrm{WL}=$ pérdida de agua en el tiempo t.

Reescribiendo la Ecuación 5 se obtiene la Ecuación 6:

$\mathrm{WL}=\mathrm{A}+\mathrm{B} \mathrm{t}^{1 / 2}$

en que:

$$
B=2 \mathrm{WL}_{\infty}\left(\frac{\mathrm{D}}{\pi \mathrm{l}^{2}}\right)^{1 / 2}
$$

A: ordenada al origen

El segundo modelo aplicado fue la ecuación empírica propuesta por Peleg (1988), la Ecuación 7, que describe las curvas que se acercan a un equilibrio asintótico. Es un modelo no-exponencial de dos parámetros:

$$
M(t)=M_{i} \pm \frac{t}{k_{1}+k_{2} t}
$$

en que:

$\mathrm{M}_{\mathrm{i}}=$ humedad inicial

$\mathrm{M}(\mathrm{t})=$ humedad al tiempo $\mathrm{t}$

$\mathrm{k}_{1} \mathrm{y} \mathrm{k}_{2}=$ constantes

Teniendo en cuenta la Ecuación 4 y la Ecuación 7 se puede escribir en términos de WL, obteniendo la Ecuación 8:

$$
\mathrm{WL}=\frac{\mathrm{t}}{\frac{\mathrm{W}_{\mathrm{i}}}{100}\left(\mathrm{k}_{1}+\mathrm{k}_{2} \mathrm{t}\right)}
$$

Similar ecuación se puede escribir para la ganancia de sólidos (SG) y para la pérdida de peso (WR) Ecuaciones 9 y 10.

$$
\begin{aligned}
& S G=\frac{t}{\frac{W_{i}}{100}\left(k_{1}+k_{2} t\right)} \\
& W R=\frac{t}{\frac{W_{i}}{100}\left(k_{1}+k_{2} t\right)}
\end{aligned}
$$


Para el modelado matemático de los datos experimentales se utilizó el software "Statictic" (versión 6.0). La calidad del ajuste entre los datos experimentales y los valores predichos por las ecuaciones de Hawkes y Flink (1978) y Peleg se analizaron a través del coeficiente de correlación $\left(\mathrm{R}^{2}\right)$.

\section{Resultados y discusión}

Los resultados obtenidos para ganancia de sólidos (SG) y pérdida de agua (WL) en muestras deshidratadas osmóticamente en solución de sacarosa al 40\%, se muestran en las Figuras 1 y 2. Se observan también, las representaciones obtenidas a partir del ajuste de los datos experimentales a la ecuación de Peleg.

La mayor pérdida de agua ocurrió dentro de los primeros 60 minutos de proceso, a partir de los cuales un aumento de tiempo no produjo cambios apreciables en el contenido de agua de las muestras, lográndose a partir de los 90 minutos, la estabilización del sistema. El valor de equilibrio fue de 68,8 g.100 g-1 MF. Estos resultados son coincidentes con lo

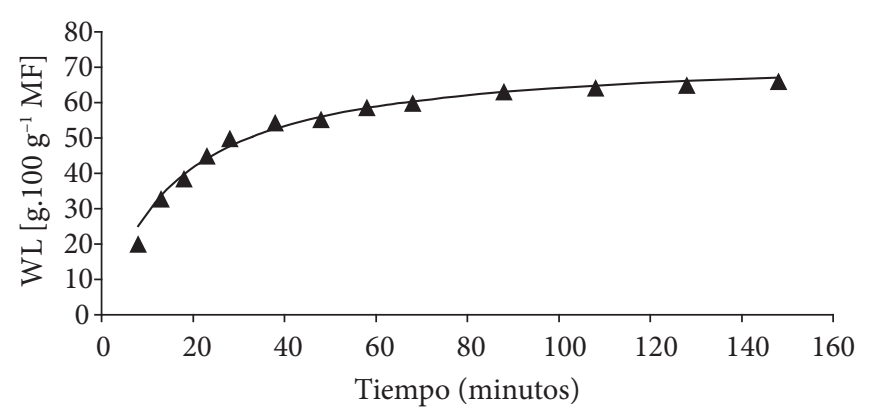

$\Delta$ Valores experimentales

— Valores modelados utilizando la ecuación de Peleg

Figura 1. Pérdida de agua de Yacón deshidratado osmóticamente en solución de sacarosa $40 \% \mathrm{p} / \mathrm{p}$.

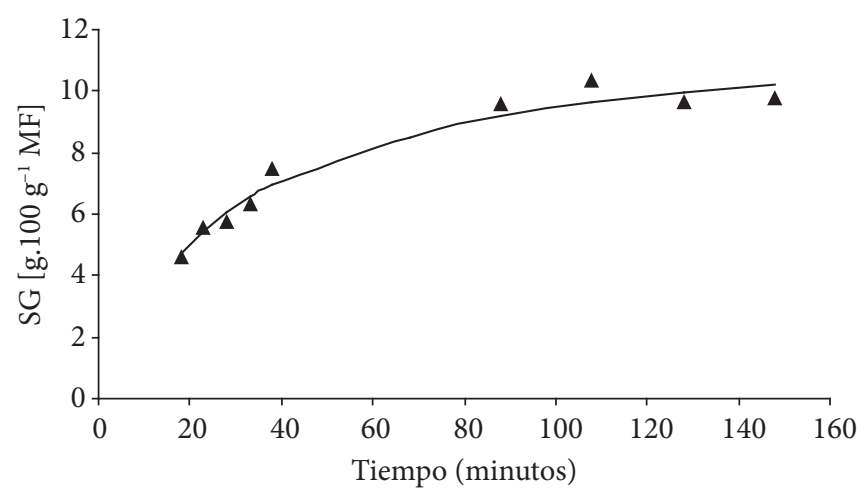

A Valores experimentales

— Valores modelados utilizando la ecuación de Peleg

Figura 2. Ganancia de sólidos de Yacón deshidratado osmóticamente en solución de sacarosa $40 \% \mathrm{p} / \mathrm{p}$. encontrado en otras matrices alimentarias (LAZARIDES; KATSANIDIS; NICKOLAIDIS, 1995; CHAFER et al., 2001; GIRALDO et al., 2003) aunque difieren de algunos trabajos (EL-AQUAR; MURR, 2003).

La ganancia de sólidos aumenta rápidamente en los primeros 60 minutos y luego sigue creciendo regularmente hasta alcanzar un valor de 9,5 g.100 g-1 MF. Estos resultados concuerdan con los encontrados por otros autores (KOWALSKA; LENART, 2001; PALOU et al., 1993).

Al cabo de aproximadamente tres horas de proceso se obtuvo una pérdida de peso de $65,84 \mathrm{~g} .100 \mathrm{~g}^{-1} \mathrm{MF}$, valor superior al reportado para otras matrices (PANADES et al., 2006; KAYMAK-ERTEKIN; SULTANO, 2000).

Las ecuaciones de Peleg y Hawkes y Flink (1978) fueron usadas para modelar los datos experimentales. De acuerdo a los resultados obtenidos, el coeficiente de difusión del proceso varía con el contenido de humedad de las muestras. Eso puede aproximarse a un proceso con dos períodos diferentes descriptos matemáticamente por líneas con pendientes diferentes, es decir, representados por coeficientes de difusión distintos para cada período. Los parámetros calculados por regresión lineal para el modelo de Hawkes y Flink (1978) se muestran en la Tabla 1.

Esto coincide con lo reportado por Palou et al. (1994), al estudiar papaya. Se puede observar que el coeficiente D es mayor para el primer período coincidentemente con los mayores valores de WL, como era de esperar.

El valor D obtenido para todo el proceso muestra semejanza con el reportado por varios autores (EL-AQUAR; MURR, 2003; KAYMAK-ERTEKIN; SULTANO, 2000), y es mayor que el reportado en otras matrices, por ejemplo, para pera.

La ecuación de Hawkes y Flink ajustó adecuadamente los datos, concordantemente con otras matrices (HAWKES; FLINK, 1978; LAZARIDES; KATSANIDIS; NICKOLAIDIS, 1995; EL-AQUAR; MURR, 2003; MAGEL; HASSABALLAH; MURPHY, 1983; RAOULT-WACK; LENART; GUILBERT, 1992). De acuerdo al análisis estadístico realizado, los valores son significativos al $0,5 \%$ con lo cual, dado que los parámetros de ajuste varían entre 0,9675 y 0,9862 se puede afirmar que ambos modelos son apropiados (ERLE; SCHUBERT, 2001; PONTING et al., 1966).

El valor de equilibrio de pérdida de agua obtenida (aproximadamente de 70\%) es similar a lo obtenido en otros productos por otros autores (EL-AQUAR; MURR, 2003; KAYMAK-ERTEKIN; SULTANO, 2000).

Los coeficientes de la ecuación de Peleg y los parámetros de ajuste se muestran en la Tabla 2. En la bibliografía se muestran valores de $\mathrm{k} 1$ en el rango entre 10 y 200 y valores de $\mathrm{k} 2$ entre 0,015 y 3 para la pérdida de agua; mientras que los valores de

Tabla 1. Parámetros de ajuste según el modelo de Hawkes y Flink.

\begin{tabular}{cccc}
\hline & $\mathrm{B}$ & $\mathrm{D}\left[\mathrm{cm}^{2} / \mathrm{s}\right]$ & $\mathrm{R}^{2}$ \\
\hline 1 $^{\circ}$ Período & 7,672 & $3,2008 \mathrm{E}-6$ & 0,9813 \\
2 $^{\circ}$ Período & 1,8404 & $1,84192 \mathrm{E}-7$ & 0,9038 \\
\hline
\end{tabular}


k1 oscilan entre 14 y 317 y los de k2 entre 0,016 y 10 para la ganancia de sólidos (SACCHETTI; GIANOTTI; DALLA ROSA, 2001; PALOU et al., 1994). La ecuación de Peleg proporciona un modelado más adecuado para todo el proceso debido a que se obtuvieron mejores resultados de $\mathrm{R}^{2}$ (MOREIRA AZOUBEL; MURR, 2004).

Por último, en la Tabla 3 se observa que la velocidad de pérdida de agua es mayor que la de ganancia de sólidos, obteniéndose una pérdida neta de peso. Esto coincide con lo reportado en la bibliografía (KOWALSKA; LENART, 2001; LAZARIDES; KATSANIDIS; NICKOLAIDIS, 1995; EL-AQUAR; MURR, 2003; PETROTOS; LAZARIDES, 2001; RAOULT-WACK; LENART; GUILBERT, 1992).

Al cabo de los primeros 60 minutos la fuerza impulsora para la transferencia de masa disminuyó a causa de la concentración de azúcar en las capas superficiales del alimento (LAZARIDES; KATSANIDIS; NICKOLAIDIS, 1995; RAOULT-WACK; LENART; GUILBERT, 1992; MURR, 2003) que actuó como una barrera a la transferencia. El efecto de dilución de la solución en las capas adyacentes fue disminuido por el efecto de la agitación (HAWKES; FLINK, 1978).

\section{Conclusión}

La mayor parte de la pérdida de agua se obtuvo dentro de los primeros 60 minutos, alcanzando un valor de $64,04 \mathrm{~g} .100 \mathrm{~g}^{-1}$ MF, a ese tiempo.

La ganancia de sólidos fue mayor en los primeros 60 minutos alcanzando un valor de 6,9 g.100 g-1 MF.

Las muestras se estabilizaron alrededor de los 90 minutos de proceso. El modelo difusional de Fick aplicado resultó adecuado.

El coeficiente de difusión varía con el contenido de humedad, pudiéndose aproximar a dos períodos difusionales durante el proceso, caracterizados por distintos coeficientes de difusión, obteniéndose un coeficiente global de difusión igual a 1,5231-E- $6 \mathrm{~cm}^{2} / \mathrm{s}$. y un $\mathrm{R}^{2}$ de 0,9199 . El modelo empírico de Peleg modeló adecuadamente los resultados experimentales lográndose una mejor aproximación a los datos experimentales, para todo el proceso.

Tabla 2. Coeficientes y parámetros de ajuste de la ecuación de Peleg.

\begin{tabular}{cccc}
\hline Parámetro & $\mathrm{k} 1$ & $\mathrm{k} 2$ & $\mathrm{R}^{2}$ \\
\hline WL & $8,2 \pm 0,1$ & $0,53 \pm 0,06$ & 0,9862 \\
SG & $234 \pm 8$ & $2,6 \pm 0,5$ & 0,9837 \\
WR & $5 \pm 1$ & $1,2 \pm 0,1$ & 0,9675 \\
\hline
\end{tabular}

Tabla 3. Velocidades de transferencia de masa durante las dos primeras etapas del proceso.

\begin{tabular}{ccc}
\hline \multirow{1}{*}{ Parámetro } & \multicolumn{2}{c}{ Velocidad $\mathrm{g} \cdot 100 \mathrm{~g}^{-1} \mathrm{MF} \times$ minutos } \\
\cline { 2 - 3 } & $\mathrm{t}=30$ minutos & $\mathrm{t}=60$ minutos \\
\hline Ganancia de sólidos & 0,1772 & 0,1013 \\
Pérdida de agua & 1,642 & 0,9418 \\
\hline
\end{tabular}

\section{Referencias bibliográficas}

CHAFER, M. et al. Kinetics of osmotic dehydration in orange and mandarin peels. J. Food Process Eng., v. 24, n. 4, p. 273-289, 2001.

CONWAY, J. et al. Mass transfer considerations in the osmotic dehydration of apples. Inst. Food Sci. Technology, v. 16, n. 1, p. 025-029, 1983.

EL-AQUAR, A. A.; MURR, F. E. X. Estudio e modelagem da cinética de desidratacâo osmótica do mamâo formosa (papaya L.). Ciencia y Tecnología Alimentaria, v. 23, n. 1, p. 69-75, 2003.

ERLE, U.; SCHUBERT, H. Combined osmotic and microwave-vacuum dehydration of apples and strawberries, J.Food Eng, v. 49, n. 2-3, p. 193-199, 2001.

GIRALDO, G. et al. Influence of sucrose solution concentration on kinetics and yield during osmotic dehydration of mango. J. of Food Eng, v. 58, n. 1, p. 33-43, 2003.

HAWKES J.; FLINK, J. M. Osmotic Concentration on fruit slice prior to freeze dehydration. J. of Food Proces and Preserv, v. 2, p. 265-284, 1978.

HERMANN, M.; FREIRE, I.; PAZOS, C. Compositional diversity of the yacon storage root. In: Impact on a changing world: Program report 1997-98. Lima, Perú: International Potato Center (CIP), 1999. p. 425-432.

KAYMAK-ERTEKIN, F.; SULTANO, M. Modeling of mass transfer during osmotic dehydration of apples. J. of Food Eng, v. 46, n. 4, p. 243-250, 2000.

KOWALSKA, H.; LENART, A. Mass exchange during osmotic pretreatment of vegetables. J. of Food Eng., v. 49, n. 2-3, p. 137-140, 2001.

LAZARIDES, H. N.; KATSANIDIS, E.; NICKOLAIDIS, A. Mass Transfer Kinetics during osmotic preconcentration aiming at minimal solid uptake. J. Food Eng., v. 25, n. 2, p. 151-166, 1995.

LEWICKI PIOTR, P. Effect of pre-drying treatment, drying and rehydration on plant tissue properties, J. Food Properties, v. 1, n. 1, p. 1-22, 1998.

MAGEE, T. R. A.; HASSABALLAH, A. A.; MURPHY, W. R. Internal mass transfer during osmotic dehydration of apple slices in sugar solutions. Int. J. Food Sci., v. 7, n. 2, p. 147-155, 1983.

MOREIRA AZOUBEL P.; MURR, E. X. Mass transfer kinetics of osmotic dehydration of cherry tomato. J. Food Eng., v. 61, n. 3, p. 291-295, 2004.

PALOU, E. et al. Deshidratación osmótica de Papaya. Efecto de la concentración del jarabe. Revista Española de Ciencia y Tecnología de Alimentos, v. 33, n. 6, p. 621-630, 1993.

. The use of Peleg's equation to model osmotic concentration of papaya. Drying Technology, v. 12, n. 4, p. 965-978, 1994.

PANADES, G. et al. Osmotic dehydration of guava: influence of operating parameters on process kinetics. J. of Food Eng, v. 72, n. 4 , p. 383-389, 2006.

PETROTOS, K.; LAZARIDES, M. Osmotic concentration of liquid foods. J. of Food Eng., v. 49, n. 2-3, p. 201-206, 2001.

PONTING, J. D. et al. Osmotic dehydration of fruits. Food Technology, v. 20, p. 125-128, 1966.

RAOULT-WACK A. L.; LENART, A.; GUILBERT, S. Recent advances in dewatering through immersion in concentrated solutions. In: MUJUNDAR A. S. (Ed.) Drying of Solids. New York: International Science Publishers, 1992. p. 211-251. 
SACCHETTI, G.; GIANOTTI, A.; DALLA ROSA, M. Sucrose salt combined effects on mass transfer kinetics and product acceptability study on apple osmotic treatments. J.Food Eng., v. 49, n. 2-3, p. 163-173, 2001.

SALVATORI, D.; ALZAMORA, S. Structural changes and mass transfer during glucose infusion of apples affected by blanching and process variables. Drying Technology, v. 18, n. 1-2, p. 361-382, 2000.
SEMINARIO, J.; VALDERRAMA, M.; MANRIQUE, I. El Yacón: Fundamentos para el aprovechamiento de un recurso promisorio. Ed.: Centro Internacional de la papa (CIP) - Universidad de Cajamarca - Agencia Suiza para el Desarrollo y la Cooperación. Lima, Perú, 2003. 